3 Ellisen LW, Bird J, West DC et al: TAN-1, the human homolog of the Drosophila notch gene, is broken by chromosomal translocations in T lymphoblastic neoplasms. Cell 1991; 66: 649-661.

4 Maillard I, Adler SH, Pear WS: Notch and the immune system. Immunity 2003; 19: 781-791.

5 Sanchez-Irizarry C, Carpenter AC, Weng AP et al: Notch subunit heterodimerization and prevention of ligand-independent proteolytic activation depend, respectively, on a novel domain and the LNR repeats. Mol Cell Biol 2004; 24: 9265-9273.

6 Pui JC, Allman D, Xu L et al: Notch1 expression in early lymphopoiesis influences $B$ versus $T$ lineage determination. Immunity 1999; 11: 299-308.
7 Pear WS, Aster JC, Scott ML et al: Exclusive development of T cell neoplasms in mice transplanted with bone marrow expressing activated Notch alleles. J. Exp. Med. 1996; 183: 2283-2291.

8 Bellavia D, Campese AF, Alesse E et al: Constitutive activation of NF-kappaB and T-cell leukemia/lymphoma in Notch3 transgenic mice. EMBO J 2000; 19: $3337-3348$.

9 Weng AP, Nam Y, Wolfe MS et al: Growth suppression of pre-T acute lymphoblastic leukemia cells by inhibition of notch signaling. Mol Cell Biol 2003; 23: 655-664.

10 Greenwald I, Seydoux G: Analysis of gainof-function mutations of the lin-12 gene of Caenorhabditis elegans. Nature 1990; 346: 197-199.
11 Berry LW, Westlund B, Schedl T: Germ-line tumor formation caused by activation of glp-1, a Caenorhabditis elegans member of the Notch family of receptors. Development 1997; 124: 925-936.

12 Feldman BJ, Hampton T, Cleary ML: A carboxy-terminal deletion mutant of Notch1 accelerates lymphoid oncogenesis in E2A-PBX1 transgenic mice. Blood 2000; 96: 1906-1913.

13 Hoemann CD, Beaulieu N, Girard L et al: Two distinct Notch1 mutant alleles are involved in the induction of $\mathrm{T}$ - cell leukemia in c-myc transgenic mice. $\mathrm{Mol}$ Cell Biol 2000; 20: 3831-3842.

14 Zweidler-McKay PA, Pear WS: Notch and T cell malignancy. Semin Cancer Biol 2004; 14: $329-340$

Research Network

\section{EUROGLYCANET: a European network focused on congenital disorders of glycosylation}

Gert Matthijs

European Journal of Human Genetics (2005) 13, 395-397.

doi:10.1038/sj.ejhg.5201359

Published online 22 December 2004

$\mathrm{T}$ he congenital disorders of glycosylation (CDG) are an emerging group of inborn errors of metabolism. Most of these genetic defects result in severe disease, mental retardation and physical handicap. EUROGLYCANET, a European network that focuses on the advancement of research, diagnosis and treatment of CDG, was created in 1999 and has been supported by the European Commission since 2000 (see Figure 1).

In this network, clinical and basic scientists collaborate in order to identify novel types of the disease and try to promote early diagnosis. These research activities are grafted onto a central database and patient sample repository. The samples circulate among the different expert laboratories - a process for which the term 'carousel testing' was coined. The network also aims to provide the basic diagnostic tools to physicians all over Europe by establishing referral laboratories in national centres. The ultimate goal of the project is to be able to precisely diagnose all cases of CDG, to get a complete inventory of the enzymatic defects that cause protein glycosylation defects and to extend the therapeutic tools available to treat CDG.

Glycosylation is the most complex type of biomolecule modification that occurs in living organisms. ${ }^{1}$ The glycosylation pathway starts as a cotranslational process in the endoplasmic reticulum (ER). A 'standard' oligosaccharide or glycan, containing 14 sugar residues, is assembled in the ER and transferred onto the nascent protein, after which the glycoprotein is transported to the Golgi apparatus. During its transit through the Golgi, the glycan structures are gradually modified into more complex, sometimes much specialised structures. This modification process is organised like in an 'assembly line', whereby the different glycosyltransferases act in a very strict order, partly as a result of their compartmental localisation in the Golgi. This process leads to the production of thousands of different glycoproteins with a myriad of different glycan chains.

So, inborn errors of glycosylation or CDG are typically multisystem diseases, with a broad spectrum of symptoms that include mental retardation and severe developmental delay, structural abnormalities of the central nervous system, cardiac defects, malformations, hormonal dysregulation, coagulation problems and peripheral neuropathies. ${ }^{2}$ CDG causes a high morbidity and a significant mortality.

Relatively simple laboratory tests that detect abnormal glycosylation in serum proteins can be used to diagnose most patients with CDG. However, expert enzymatic, biochemical or molecular investigations are required to identify the underlying glycosylation defect. So the expert diagnostic services of the laboratories that are members of the network have 


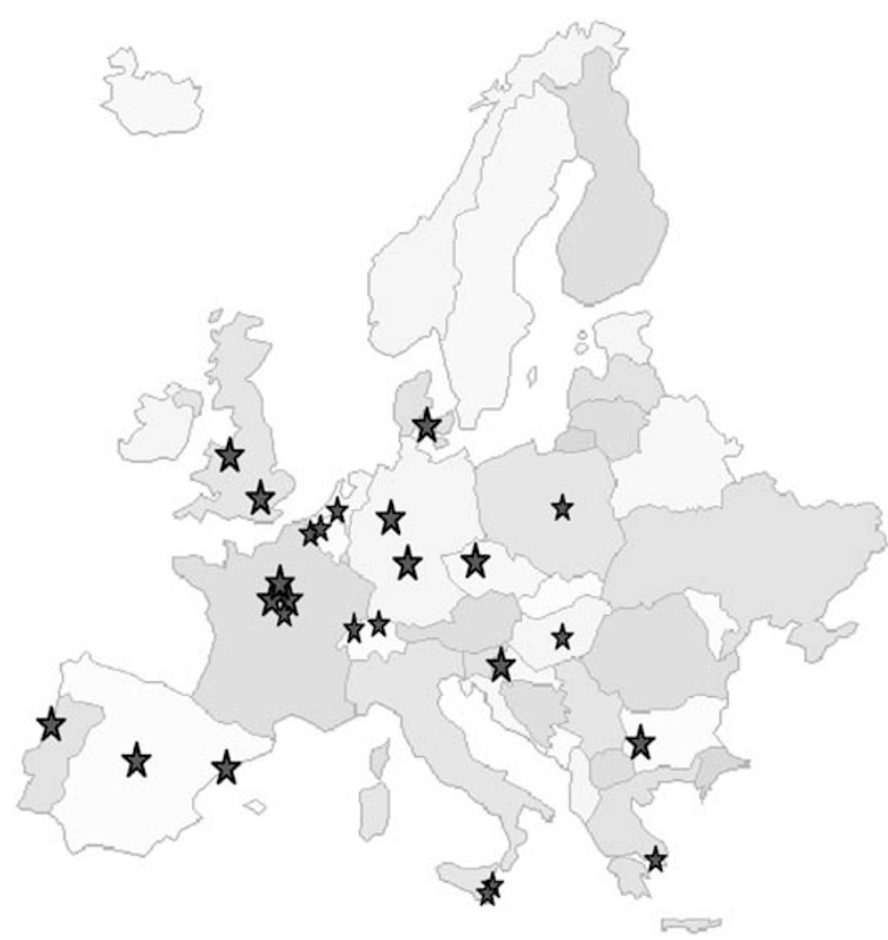

Figure 1 Geographical presentation of the network. All participants, except the two in Israel, are situated on this map. Professor Gert Matthijs, based at the Centre for Human Genetics of the University of Leuven (B), coordinates EUROGLYCANET in close collaboration with Professor Jaak Jaeken, who is at the Centre for Metabolic Diseases at the University Hospital in Leuven (B), and who described the first cases of CDG back in 1980. Other core participants in this and in the previous network are Professor Markus Aebi (Eidgenössissche Hochschule, Zürich, CH), Dr Paz Briones and Dr M Antonia Vilaseca (Institut Bioquímica Clínica, and Hospital Sant Joan de Deu, Barcelona, E), Professor Kurt von Figura (University of Göttingen, D), Professor Thierry Hennet (University of Zürich, CH), Professor Nathalie Seta (Hopital Bichat, Paris, F), Professor Emile Van Schaftingen (Christian de Duve Institute of Cellular Pathology, Brussels, B), Professor Ron Wevers (University of Nijmegen, NL), and Professor Bryan Winchester (Institute of Child Health, London, UK). In total, 27 partners from 16 countries are involved in EUROGLYCANET. The full list of participants is available on www.euroglycanet.org.

an important role in the diagnosis of CDG.

The early steps of glycosylation in the ER are perfectly conserved from yeast to human. ${ }^{3}$ The evolutionary conservation of these steps means that a wide diversity of researchers are appropriate participants in EUROGLYCANET: clinical groups, yeast geneticists (specialised in glycosylation) and molecular geneticists are all represented. However, the processing of the glycans in the Golgi is less well conserved. In this case, the analysis of the complex glycan structures is necessary for the identifi- cation of novel defects. This requires highly specialised skills and tools that are not normally available in the clinical or basic research laboratories. Thus, the network also includes glycobiologists and structural biologists, who use cutting-edge analytical systems to unravel the complexity of the structures, and, importantly, to characterise the abnormal structures in the CDG patients. The network also includes experts in biochemistry and cell biology, for the development of enzymatic assays and of cellular and animal models for these diseases. Furthermore, the clinical field is expanding, in that the defects of glycosylation now also include tissue-specific disorders, such as defects of cell signalling leading to aberrant cell growth, defects of leukocyte trafficking and congenital muscular dystrophies with migration abnormalities of the brain. ${ }^{4}$ These disorders result from very specific glycan defects, which are not readily detected in the blood, and for which other screening tools will have to be developed.

The backbone of the network is a centralised database and repository for patient data and samples. The samples are circulated among the different research laboratories, until they reach a final diagnosis. The network has also created national referral centres in different European countries, including the new member states. The idea is that the local clinicians - paediatricians, clinical geneticists, neurologists, etc. contact their national referral centre whenever they suspect a patient of having CDG. The national centres have the tools to confirm whether indeed the patient has CDG, and will then forward the information and the samples to the research network for further work-up. Owing to the European and other research funding to the different groups, these expert diagnostic services can be offered at no cost for the patients and their families. The provision of these services at no cost allows the network easy access to patients and recruitment of interesting cases. Over the years, a rich collection of 'unsolved cases' had been gathered. They are both the fruit and the basis of the intense collaboration between the groups.

The network is also committed to providing information to the public and to physicians and other professionals, offering training to expert clinicians and researchers in the field, with training courses in 2005 and 2007, and organising the next international meeting on CDG in Paris in 2007.

The European Commission supports EUROGLYCANET, which is a Co-ordination Action (CA) of the Sixth Framework programme and will be funded 
from 2005 to 2008. It is the successor and an extension of EUROGLYCAN, a network that was created in 1999, and funded under the Fifth Framework from 2000 to 2003, as a Research and Technological Development (RTD) project. European laboratories were in a particularly good position to provide a major impetus to this research, because these diseases were first identified in Europe. As a result of this collaboration, most new cases and types of CDG have also been identified in Europe.

The CA is meant to integrate research. The funding is essentially limited to coordination and integration via meetings, training courses and other types of exchanges, and in this case, also to the expert diagnostics and eventually the therapeutic trials. The network relies on other grants for the more fundamental research activities.

The challenge for this network is to keep up with the growing list of diseases in this area, and to warrant early diagnosis for this plethora of diseases. From a research standpoint, it would be interesting to see what the role of glycosylation and glycosylation defects is in the more common diseases like diabetes and neurodegenerative disorders. However, the major challenge for the (larger) clinical and basic research community will be to develop therapies for these complex diseases. One wonders for instance whether enzyme replacement would have a role in their treatment, or whether simple, pharmacological agents could be identified to bypass the deficient enzymes or boost their activity.

The merit of this network is that, within a few years, it has consolidated the European lead on clinical and fundamental research into these diseases. At the same time, it has shown that for rare diseases, the close interaction between expert clinicians and specialised researchers, together with the centrally monitored 'carousel' testing, are the key to success. This network could stand as a model for the organisation and integration of clinical and basic research for other rare diseases, in and beyond the metabolic field.

Clinicians who wish to share patient material with the network can either contact the network or the national referral centres, via www.euroglycanet. org. The network of course also accepts samples from abroad. Research groups or companies that are interested in contributing to the research in this field are invited to contact the coordinator

Professor G Matthijs is at the Center for Human Genetics, University of Leuven, Herestraat 49, Leuven B-3000, Belgium. E-mail: Gert.Matthijs@med. kuleuven.ac.be

\section{References}

1 Lowe JB, Marth JD: A genetic approach to mammalian glycan function. Annu Rev Biochem 2003; 72: 643-691.

2 Jaeken J, Matthijs G, Carchon H, Van Schaftingen E: Defects of N-glycan synthesis; In: Scriver CR, Beaudet AL, Sly WS, Valle D (eds): The metabolic and molecular bases of inherited diseases. New York: McGraw-Hill, 2001, pp 1601-1622.

3 Aebi M, Hennet T: Congenital disorders of glycosylation: genetic model systems lead the way. Trends Cell Biol 2001; 11: $136-141$.

4 Jaeken J: Komrower Lecture. Congenital disorders of glycosylation (CDG): it's all in it!. J Inherit Metab Dis 2003; 26: 99-118.

\section{Cascade Screening}

\section{Whose information is it anyway?}

Guido de Wert

European Journal of Human Genetics (2005) 13, 397-398.

doi:10.1038/sj.ejhg.5201373

Published online 26 January 2005

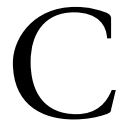
ascade screening, that is, systematically approaching relatives of patients affected by genetic disorders, is controversial. Objections include that it undermines the autonomy of relatives, as they may be (or at least feel) pressurized to participate in the program, and that it is an invasion of their privacy, more in particular their right 'not to know' that they are at risk. The validity of these objections is questionable. Firstly, much will depend upon how relatives are approached and informed: is the approach coercive or not, is the information provided stepwise, do people get time to think through the issues involved, etc. ${ }^{1}$ It is important to spell out the primary aim of a cascade screening program. Is it to contact and inform as many relatives as possible in order to enable them to make informed decisions regarding testing and possible preventive measures? Or does the program aim at testing all relatives at risk? In the latter case, the program would be at odds with the requirement of voluntary participation in the screening. Secondly, critics tend to ignore that relatives may have the right to know, conditional upon the preventive value of the information. An ethical view that focuses exclusively on relatives' right not to know does not do justice to the (possible) relatives' health and/ or reproductive interests involved and is, therefore, one dimensional. Thirdly, in traditional clinical genetics, the professional standard urges counselors to explicitly point to the possible interests of clients' relatives 'the patient is the family'. If one accepts this practice, one cannot consistently argue that cascade screening 